\title{
Single Balloon Enteroscopy-Assisted Endoscopic Retrograde Cholangiopancreatography in Patients Who Underwent a Gastrectomy with Roux-en-Y Anastomosis: Six Cases from a Single Center
}

\author{
Jae Seung Soh, Dong-Hoon Yang, Sang Soo Lee, Seohyun Lee, Jungho Bae, Jeong-Sik Byeon, Seung-Jae Myung \\ and Suk-Kyun Yang
}

Department of Gastroenterology, Asan Digestive Disease Research Institute, Asan Medical Center, University of Ulsan College of Medicine, Seoul, Korea

Patients with altered anatomy such as a Roux-en-Y anastomosis often present with various pancreaticobiliary problems requiring therapeutic intervention. However, a conventional endoscopic approach to the papilla is very difficult owing to the long afferent limb and acute angle of a Roux-en-Y anastomosis. Balloon-assisted enteroscopy can be used for endoscopic retrograde cholangiopancreatography (ERCP) in patients with altered anatomy. We experienced six cases of Roux-en-Y anastomosis with biliary problems, and attempted ERCP using single balloon enteroscopy (SBE). SBE insertion followed by replacement with a conventional endoscope was attempted in five of six patients. The papilla was successfully approached using SBE in all cases. However, therapeutic intervention was completed in only three cases because of poor maneuverability caused by postoperative adhesion. We conclude that in patients with Roux-en-Y anastomosis, the ampulla can be readily accessed with SBE, but longer dedicated accessories are necessary to improve this therapeutic intervention. Clin Endosc 2015;48:452-457

Key Words: Single balloon enteroscopy; Cholangiopancreatography, endoscopic retrograde; Anastomosis, Roux-en-Y

\section{INTRODUCTION}

Patients with a Roux-en-Y anastomosis may present with various pancreaticobiliary problems requiring endoscopic intervention. ${ }^{1}$ However, performing endoscopic retrograde cholangiopancreatography (ERCP) in these cases is very difficult because conventional endoscopes can barely reach the papilla in the long afferent limb. Recent studies have suggested that

Received: August 12, 2014 Revised: October 22, 2014

Accepted: December 16, 2014

Correspondence: Dong-Hoon Yang

Department of Gastroenterology, Asan Medical Center, University of Ulsan College of Medicine, 88 Olympic-ro 43-gil, Songpa-gu, Seoul 05505, Korea

Tel: +82-2-3010-5809, Fax: +82-2-3010-6517, E-mail: dhyang@amc.seoul.kr

(cc) This is an Open Access article distributed under the terms of the Creative Commons Attribution Non-Commercial License (http://creativecommons.org/ licenses/by-nc/3.0) which permits unrestricted non-commercial use, distribution, and reproduction in any medium, provided the original work is properly cited. balloon-assisted enteroscopy (BAE) can provide direct access to the major papilla in the afferent loop, and can therefore be used for ERCP in patients with a Roux-en-Y anastomosis. ${ }^{1,2}$ We experienced six cases involving Roux-en-Y anastomosis and biliary problems at our center, and assessed the utility of ERCP with single balloon enteroscopy (SBE) in these patients.

\section{CASE REPORTS}

A single balloon enteroscope with a length of $200 \mathrm{~cm}$, distal end diameter of $9.2 \mathrm{~mm}$, and channel diameter of $2.8 \mathrm{~mm}$ (SIF-Q260; Olympus Co., Tokyo, Japan) and an overtube (STSB1; Olympus Co.) were used to access the afferent limb in all cases. Because a routine ERCP catheter could not reach the ampulla using SBE owing to long channel length and a long ERCP catheter was not available in Korea, a self-assembled 
long catheter was used to acquire a cholangiogram in the first case. Single balloon enteroscope insertion followed by replacement with a conventional endoscope, as described previously, ${ }^{3}$ was used for ERCP in the next five cases because long accessories were not available.

\section{Case 1}

A 66-year-old man was transferred with a diagnosis of biliary sepsis. Computed tomography (CT) revealed a dilatation of the common bile duct (CBD) and a swelling of the pancreas with peripancreatic infiltration. One year previous- ly, he had undergone a total gastrectomy with Roux-en-Y anastomosis for gastric cancer. ERCP was performed using a forward-viewing esophagogastroduodenoscope, but the ampulla could not be reached. The following day, ERCP using SBE was attempted, and the papilla was successfully reached in 29 minutes. To produce a long catheter, after removing the injection needle from the colonoscopic injection catheter, the injection catheter end was conjoined with the infusion part of the ERCP catheter. Using this self-assembled long catheter, cholangiography was performed and showed mild dilation of the CBD and pancreatic duct, but no visible stone. Based on
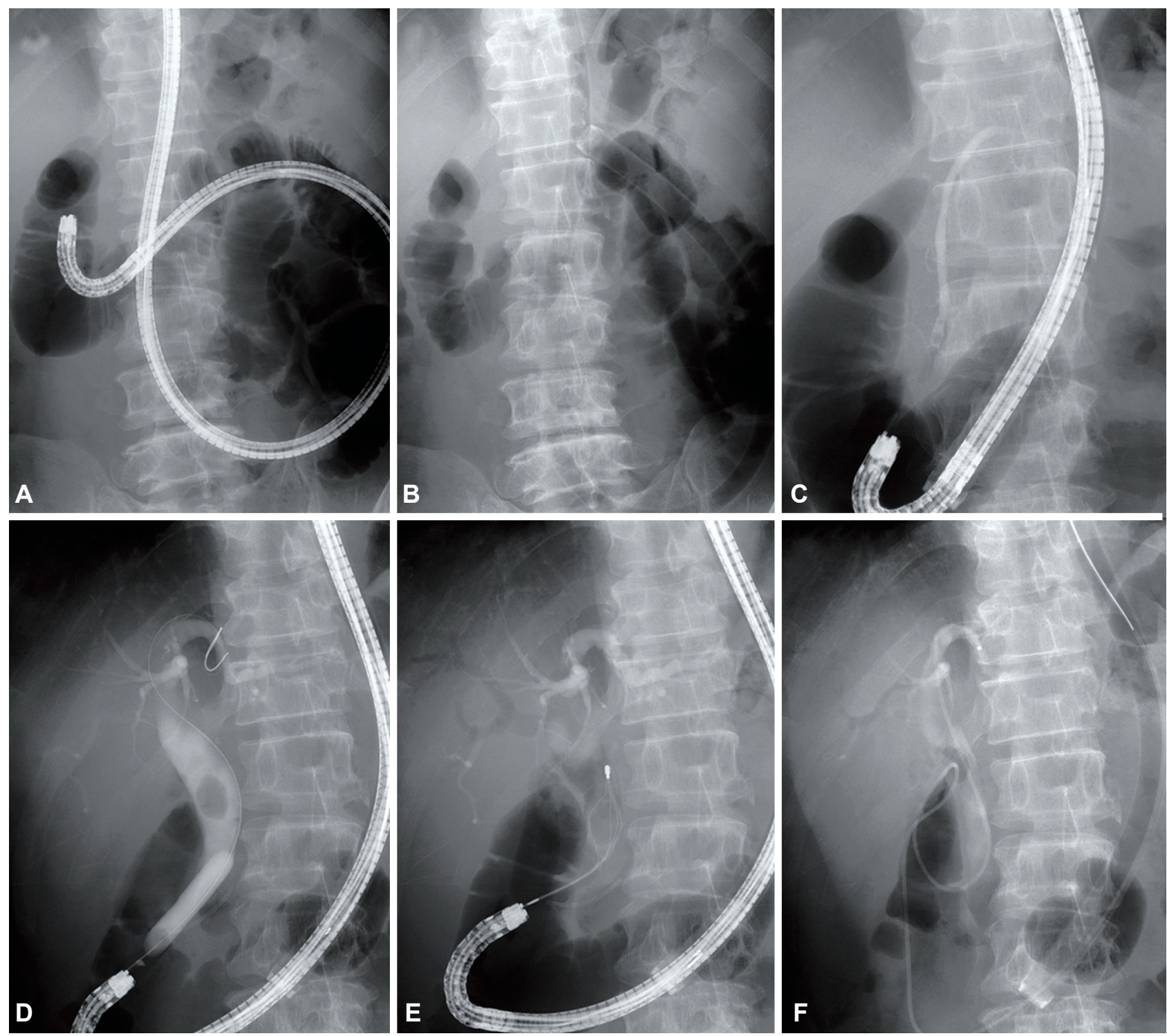

Fig. 1. A 58-year-old man who received a total gastrectomy with a Roux-en-Y anastomosis had a 17-mm brown stone removed by single balloon enteroscope (SBE)-assisted endoscopic retrograde cholangiopancreatography (ERCP). (A) Simple radiography showing the tip of the SBE reaching the end of the afferent loop. (B) The SBE was withdrawn leaving the overtube. (C) An esophagogastroduodenoscope was inserted successfully through the in-dwelling overtube. (D) A cholangiogram showing a diffuse dilatation and a 17-mm filling defect in the common bile duct. Endoscopic papillary dilatation was performed with the controlled radial expansion balloon. (E) The stone was removed using a mechanical lithotripter and a retrieval balloon. (F) An endoscopic nasobiliary drain was inserted and ERCP was completed successfully. 
these findings, we assessed that the biliary stone had already been passed. To improve pancreatitis, a plastic stent ( $5 \mathrm{Fr}, 3$ $\mathrm{cm}$, Cook Medical Inc., Bloomington, IN, USA) was inserted into the pancreatic duct. The patient was discharged without complications 4 days later.

\section{Case 2}

A 58-year-old man who had undergone a total gastrectomy with Roux-en-Y anastomosis for gastric cancer visited our emergency department with abdominal pain and fever. A CT scan revealed a $17-\mathrm{mm}$ stone obstructing the CBD. A single balloon enteroscope was inserted, and the papilla was reached after 14 minutes. After shortening the small bowel, the enteroscope was withdrawn, leaving the overtube. An esophagogastroduodenoscope was successfully inserted through the in-dwelling overtube, and the standard accessories for ERCP could then be used. Cholangiography showed a diffuse dilatation and a $17-\mathrm{mm}$ filling defect in the CBD. After dilating the papilla with a controlled radial expansion (CRE) balloon (Boston Scientific, Natick, MA, USA), a brown stone was removed using a mechanical lithotripter and retrieval balloon

(Fig. 1). The patient experienced mild post-ERCP pancreatitis presenting with abdominal pain, a serum amylase level of 1,380 IU/L, and a serum lipase level of 2,969 IU/L, but recovered with conservative treatment. He was discharged 6 days after ERCP without event.

\section{Case 3}

A 73-year-old man who had undergone a total gastrectomy with Roux-en-Y anastomosis for gastric cancer was admitted for removal of a 12-mm CBD stone. Although the ampulla was reached using SBE in 38 minutes, the small bowel was not shortened effectively, and a conventional endoscope could therefore not pass through the overtube. The next best treatment option was to leave the guide wires in the afferent limb and introduce a pediatric colonoscope equipped with a cap along these wires, but this approach also failed. Consequently, percutaneous transhepatic choledochoscopy (PTCS) was performed.

\section{Case 4}

A 72-year-old woman visited the emergency department with epigastric pain and fever. A CT scan suggested CBD stones and cholangitis. Four years previously, she had under-
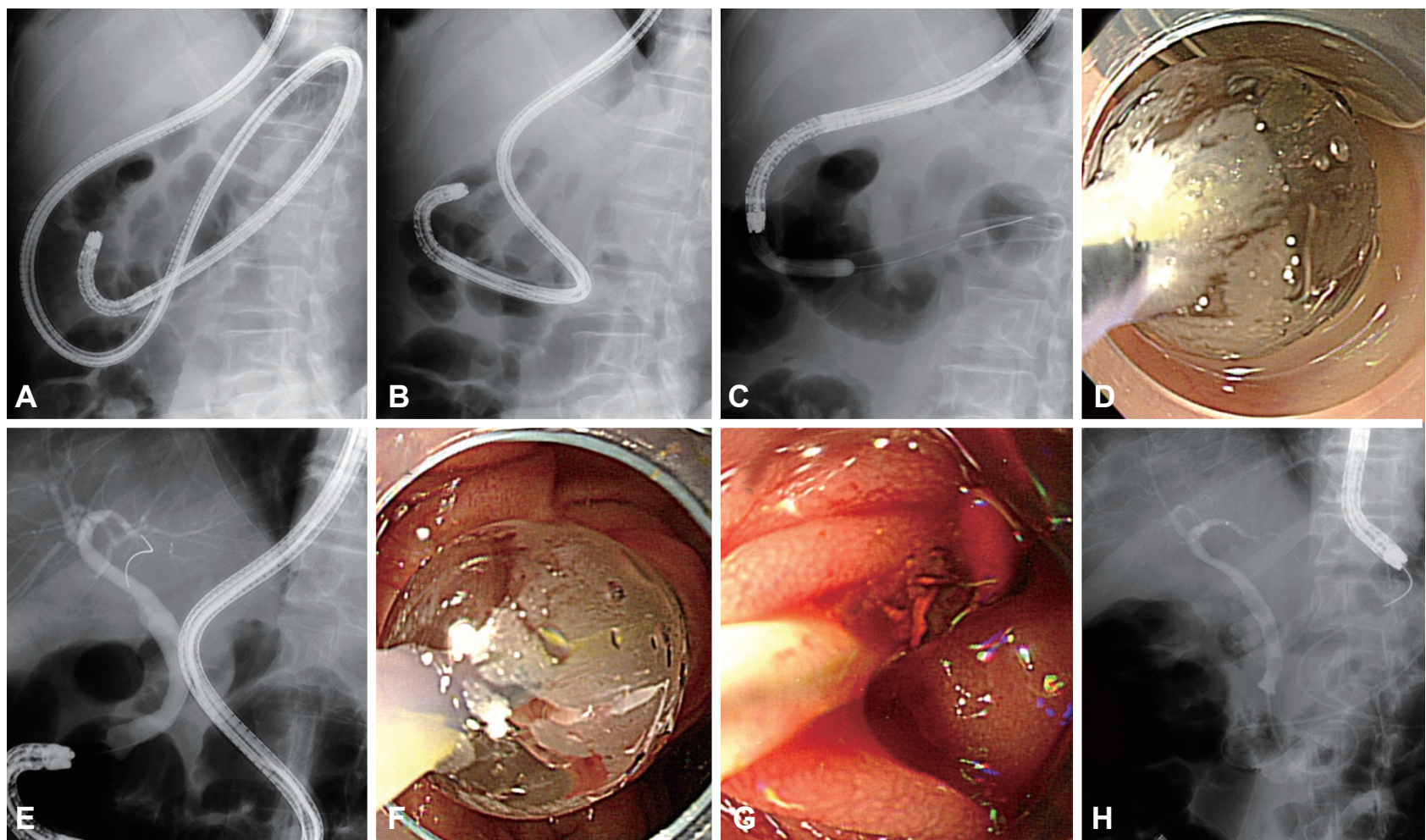

Fig. 2. A 78-year-old man who received a prior total gastrectomy with a Roux-en-Y anastomosis had small brown stones successfully removed by single balloon enteroscope (SBE)-assisted endoscopic retrograde cholangiopancreatography (ERCP). (A) The SBE reached the afferent loop. (B) The enteroscope was $80 \mathrm{~cm}$ in length. (C) While inserting the esophagogastroduodenoscope, an acute angulation was made $25 \mathrm{~cm}$ distal to the overtube. After the collapsed site was dilated by the controlled radial expansion (CRE) balloon, a conventional endoscope could be inserted into the overtube. (D) Endoscopic view; the CRE balloon dilated the acute angulation. (E) Dilatation of the common bile duct was noted on the cholangiogram. (F) Endoscopic papillary dilatation was performed with a CRE balloon. (G) Small brown stones were removed using the retrieval balloon. $(\mathrm{H})$ An endoscopic nasobiliary drainage was inserted and ERCP was completed successfully. 
gone a total gastrectomy with Roux-en-Y anastomosis for gastric cancer. Jejunojejunostomy was performed by sideto-side anastomosis, and an extremely acute angulation was formed between the afferent and efferent limbs. Access to the papilla using SBE was therefore quite difficult, and 70 minutes were required. Replacing a conventional scope through the overtube was impossible owing to the angulation at the Rouxen-Y anastomosis. A CRE balloon was used at the collapsed site of the angulation, and the conventional scope was eventually passed. Although the conventional scope could reach the papilla, cannulation was not successful. Therefore, the CBD stones were removed by PTCS.

\section{Case 5}

A 69-year-old woman who had undergone a distal gastrectomy with Roux-en-Y anastomosis for gastric cancer visited the emergency room with abdominal pain, fever, and hypotension. A CT scan revealed calculous cholecystitis and a distal CBD stone. Percutaneous gallbladder drainage was performed initially, and SBE-assisted ERCP was attempted 2 days later. After accessing the papilla, the enteroscope was shortened and replaced with a pediatric colonoscope through the overtube. Cholangiography revealed a $12-\mathrm{mm}$ filling defect. However, removal of the CBD stone failed because of poor maneuverability. The patient was eventually treated by percutaneous transhepatic papillary balloon dilation.

\section{Case 6}

A 78-year-old man was transferred with a diagnosis of cholangitis. Three years prior, he had undergone a total gastrectomy with Roux-en-Y anastomosis for gastric cancer. A single balloon enteroscope was inserted into the afferent loop. After shortening of the small bowel, the enteroscope was retrieved, but the remaining overtube was collapsed because of the acute angulation of the small bowel. A CRE balloon was used to dilate the collapsed site, and a conventional endoscope could then be pushed into the overtube. Small brown stones were successfully removed by ERCP (Fig. 2).

\section{DISCUSSION}

Patients who have undergone Roux-en-Y anastomosis sometimes present with pancreaticobiliary problems. Because ERCP in these patients is extremely difficult owing to their anatomical complexity, including a long afferent loop or difficulty in maneuvering, more invasive interventions such as percutaneous transhepatic biliary drainage, PTCS, or surgery are often necessary. ${ }^{4}$ ERCP using a forward-viewing endoscope such as a pediatric colonoscope might be the first choice treatment in patients with altered anatomy. ${ }^{5,6}$ BAE provides direct access to the major papilla located in the long afferent loop, and therefore, enables ERCP to be performed in patients who have had a Roux-en-Y anastomosis. ERCP using double balloon enteroscopy (DBE) is an innovative technique because of its ability to enable ERCP-related interventions in patients with altered anatomy after surgery.- ${ }^{7-9}$ SBE has the advantages of a shorter preparation time and better maneuverability compared with DBE. ERCP using SBE was first reported in $2008,{ }^{10}$ and several case series of SBE-ERCP in patients with Roux-en-Y anastomosis have been published. ${ }^{11-13}$ Two studies that compared SBE-ERCP with forward-viewing endoscope (including colonoscope)-assisted $\mathrm{ERCP}^{14}$ or DBE-ERCP ${ }^{15}$ reported that SBE was not inferior in accessing the papilla or performing therapeutic ERCP in patients with a Roux-en-Y anastomosis. Table 1 showed the characteristics and diagnostic and therapeutic success rates of ERCP using colonoscopy, DBE, and SBE.

Table 1. Characteristics and Diagnostic and Therapeutic Success Rate for Endoscopic Retrograde Cholangiopancreatography of Colonoscopy, DBE, and SBE Performed in Patients with Altered Anatomy

\begin{tabular}{|c|c|c|c|c|c|c|}
\hline & $\begin{array}{l}\text { Working } \\
\text { length, } \mathrm{cm}\end{array}$ & $\begin{array}{c}\text { Distal end } \\
\text { diameter, } \\
\text { mm }\end{array}$ & $\begin{array}{c}\text { Instrumental } \\
\text { channel } \\
\text { diameter, } \mathrm{mm}\end{array}$ & $\begin{array}{c}\text { Diagnostic } \\
\text { success rate, } \\
\%^{\text {a) }}\end{array}$ & $\begin{array}{c}\text { Therapeutic } \\
\text { success rate, } \\
\left.\%^{b}\right)\end{array}$ & Comment \\
\hline Colonoscope $e^{5,6}$ & 168 & 11.3 & 3.2 & $67-82$ & $86-100$ & $\begin{array}{l}\text { Reasonable first choice in short-limb } \\
\text { Roux-en-Y }\end{array}$ \\
\hline $\mathrm{DBE}^{7-9}$ & 200 & 9.4 & 2.8 & $83-93$ & $58-92$ & $\begin{array}{l}\text { Longer length than colonoscopy, Balloon } \\
\text { and overtube system available }\end{array}$ \\
\hline $\mathrm{SBE}^{11-15}$ & 200 & 9.2 & 2.8 & $70-80$ & $58-91$ & $\begin{array}{l}\text { A shorter preparation time and better } \\
\text { maneuverability compared with DBE }\end{array}$ \\
\hline
\end{tabular}

Pediatric colonoscope (PCF-140L; Olympus Co.), DBE (EN-450T5; Fujinon), and SBE (SIF-Q180 or SIF-Q260; Olympus Co.) were used in these studies.

DBE, double balloon enteroscopy; SBE, single balloon enteroscopy.

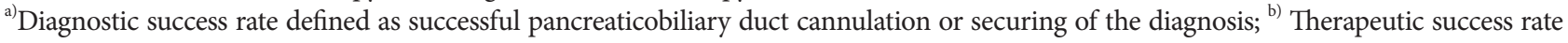
defined as successfully accomplishing the endoscopic therapy. 


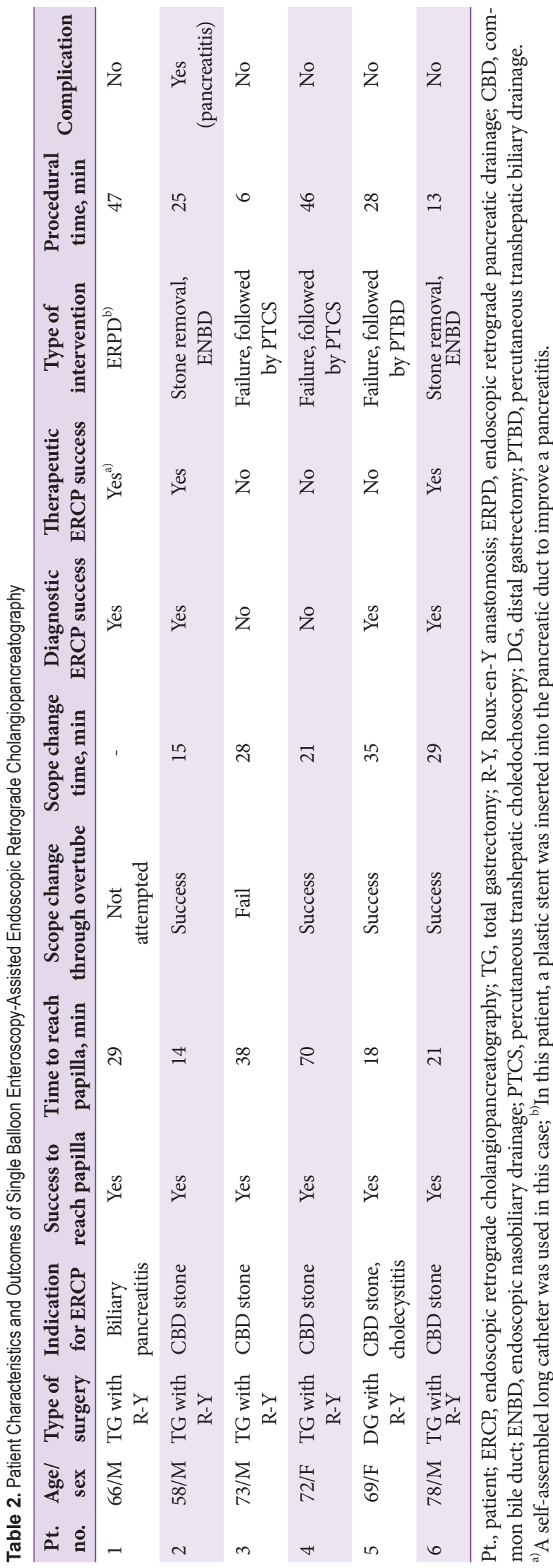

Based on the aforementioned studies, we attempted ERCP using SBE in patients with Roux-en-Y anastomosis due to biliary problems. Although the papilla was reached using SBE in all patients, a cholangiography that secured the diagnosis was obtained in four of six cases, and therapeutic intervention succeeded in only three (Table 2). Technical inexperience might have influenced this relatively low therapeutic success rate. The absence of an elevator in the enteroscope or conventional endoscope also limited maneuverability during ERCP. Because long accessories compatible with an enteroscope were not available, we chose to perform single balloon enteroscope insertion followed by replacement with a conventional endoscope, which enabled us to use standard accessories. Unlike the report of Itoi et al. ${ }^{3}$ that first described such an exchange method and a success rate of $72.1 \%(8 / 11)$ in patients with Roux-en-Y anastomosis, our success rate was relatively low. However, a direct comparison between the results of this study and those obtained in the study by Itoi et al. ${ }^{3}$ is not possible owing to differences in surgical methods, the degree of postoperative intra-abdominal adhesion, and the endoscopist's experience. To perform ERCP in patients with altered anatomy, various other methods including short type $\mathrm{SBE},{ }^{16}$ short type $\mathrm{DBE},{ }^{17}$ the endoscopic ultrasound-guided antegrade approach, ${ }^{18}$ and cap-assisted approach ${ }^{19}$ have been introduced with success rates ranging from $70 \%$ to $98.5 \%$.

Complications such as bleeding, infection, and perforation can occur with ERCP using BAE owing to long procedure times and the level of difficulty involved. In our study, the mean procedural time was 80.5 minutes (range, 54 to 137). However, only one patient experienced mild pancreatitis after ERCP despite the relatively long procedure time. According to earlier reports on SBE-ERCP, procedure-related complications such as pancreatitis or pseudocyst formation occur in $0 \%$ to $18.2 \%$ of cases. ${ }^{3,11-15}$

In conclusion, ERCP using BAE is a feasible and useful intervention in patients with Roux-en-Y anastomosis. However, to improve the success rate of this method, technical advances are needed, including the development of long accessories that are compatible with the enteroscope.

\section{Conflicts of Interest}

The authors have no financial conflicts of interest.

\section{REFERENCES}

1. Koornstra JJ, Fry L, Mönkemüller K. ERCP with the balloon-assisted enteroscopy technique: a systematic review. Dig Dis 2008;26:324-329.

2. Moreels TG. Altered anatomy: enteroscopy and ERCP procedure. Best Pract Res Clin Gastroenterol 2012;26:347-357.

3. Itoi T, Ishii K, Sofuni A, et al. Single-balloon enteroscopy-assisted ERCP in patients with Billroth II gastrectomy or Roux-en-Y anastomosis (with 
video). Am J Gastroenterol 2010;105:93-99.

4. Hintze RE, Adler A, Veltzke W, Abou-Rebyeh H. Endoscopic access to the papilla of Vater for endoscopic retrograde cholangiopancreatography in patients with billroth II or Roux-en-Y gastrojejunostomy. Endoscopy 1997;29:69-73.

5. Elton E, Hanson BL, Qaseem T, Howell DA. Diagnostic and therapeutic ERCP using an enteroscope and a pediatric colonoscope in long-limb surgical bypass patients. Gastrointest Endosc 1998;47:62-67.

6. Wright BE, Cass OW, Freeman ML. ERCP in patients with long-limb Roux-en-Y gastrojejunostomy and intact papilla. Gastrointest Endosc 2002;56:225-232.

7. Emmett DS, Mallat DB. Double-balloon ERCP in patients who have undergone Roux-en-Y surgery: a case series. Gastrointest Endosc 2007;66:1038-1041.

8. Mönkemüller K, Fry LC, Bellutti M, Neumann H, Malfertheiner P. ERCP with the double balloon enteroscope in patients with Roux-en-Y anastomosis. Surg Endosc 2009;23:1961-1967.

9. Parlak E, Ciçek B, Dişibeyaz S, et al. Endoscopic retrograde cholangiography by double balloon enteroscopy in patients with Roux-en-Y hepaticojejunostomy. Surg Endosc 2010;24:466-470.

10. Mönkemüller K, Fry LC, Bellutti M, Neumann H, Malfertheiner P. ERCP using single-balloon instead of double-balloon enteroscopy in patients with Roux-en-Y anastomosis. Endoscopy 2008;40 Suppl 2:E19-E20

11. Kianička B, Lata J, Novotný I, Dítě P, Vaníček J. Single balloon enteroscopy for endoscopic retrograde cholangiography in patients with Rouxen-Y hepaticojejuno anastomosis. World J Gastroenterol 2013;19:80478055.

12. Saleem A, Baron TH, Gostout CJ, et al. Endoscopic retrograde cholan- giopancreatography using a single-balloon enteroscope in patients with altered Roux-en-Y anatomy. Endoscopy 2010;42:656-660.

13. Tomizawa Y, Sullivan CT, Gelrud A. Single balloon enteroscopy (SBE) assisted therapeutic endoscopic retrograde cholangiopancreatography (ERCP) in patients with roux-en-y anastomosis. Dig Dis Sci 2014;59:465-470.

14. Kawamura T, Mandai K, Uno K, Yasuda K. Does single-balloon enteroscopy contribute to successful endoscopic retrograde cholangiopancreatography in patients with surgically altered gastrointestinal anatomy? ISRN Gastroenterol 2013;2013:214958.

15. Moreels TG, Pelckmans PA. Comparison between double-balloon and single-balloon enteroscopy in therapeutic ERC after Roux-en-Y entero-enteric anastomosis. World J Gastrointest Endosc 2010;2:314-317.

16. Kawamura T, Uno K, Suzuki A, et al. Clinical usefulness of a short-type, prototype single-balloon enteroscope for endoscopic retrograde cholangiopancreatography in patients with altered gastrointestinal anatomy: preliminary experiences. Dig Endosc 2015;27:82-86.

17. Siddiqui AA, Chaaya A, Shelton C, et al. Utility of the short double-balloon enteroscope to perform pancreaticobiliary interventions in patients with surgically altered anatomy in a US multicenter study. Dig Dis Sci 2013;58:858-864.

18. Itoi T, Sofuni A, Tsuchiya T, Ijima M, Iwashita T. Endoscopic ultrasonography-guided transhepatic antegrade stone removal in patients with surgically altered anatomy: case series and technical review (with videos). J Hepatobiliary Pancreat Sci 2014;21:E86-E93.

19. Ki HS, Park $\mathrm{CH}$, Jun $\mathrm{CH}$, et al. Feasibility of cap-assisted endoscopic retrograde cholangiopancreatography in patients with altered gastrointestinal anatomy. Gut Liver 2015;9:109-112. 